\title{
Lesion load assessment among multiple sclerosis patient using DIR, FLAIR, and T2WI sequences
}

\author{
Abdullah Dhaifallah Almutairi', Hasyma Abu Hassan', Subapriya Suppiah', Othman I. Alomair², \\ Abdulbaset Alshoaibi ${ }^{3}$, Hajab Almutairi ${ }^{4}$ and Rozi Mahmud ${ }^{1 *}$
}

\begin{abstract}
Background: Magnetic resonance imaging (MRI) is one of the diagnostic imaging modalities employing in lesion detection in neurological disorders such as multiple sclerosis (MS). Advances in MRI techniques such as double inversion recovery (DIR) made it more sensitive to distinguish lesions in the brain. To investigate the lesion load on different anatomical regions of the brain with MS using DIR, fluid attenuated inversion recovery (FLAIR) and T2weighted imaging (T2WI) sequences. A total of $97 \mathrm{MS}$ patients were included in our retrospective study, confirmed by neurologist. The patients were randomly selected from the major hospital in Saudi Arabia. All images were obtained using 3T Scanner (Siemens Skyra). The images from the DIR, FLAIR, and T2WI sequence were compared on axial planes with identical anatomic position and the number of lesions was assigned to their anatomical region.

Results: Comparing the lesion load measurement at various brain anatomical regions showed a significant difference among those three methods ( $p<0.05$ ).

Conclusion: DIR is a valuable MRI sequence for better delineation, greater contrast measurements and the increasing total number of MS lesions in MRI, compared with FLAIR, and T2WI and DIR revealed more intracortical lesions as well; therefore, in MS patients, it is recommended to add DIR sequence in daily routine imaging sequences.
\end{abstract}

Keywords: Double inversion recovery, FLAIR, T2WI, MRI, Multiple sclerosis

\section{Background}

Multiple sclerosis (MS) is an autoimmune inflammatory disease of the central nervous system (CNS), resulting to neurological disorder in both the youth and the elderly in the worldwide and accounts for about $1.5 \%$ of neurological abnormalities, associated with a progressive physical damage $[1,2]$. The disease affects the calloso-septal interface, periventricular white matter, cerebellum, and brain stem $[3,4]$. Several studies revealed that most MS lesion load histologically are on the cerebral cortex specifically the gray matter and white mater interface [5],

\footnotetext{
* Correspondence: rozi@upm.edu.my

${ }^{1}$ Department of Imaging, Faculty of Medicine and Health Sciences, Universiti

Putra Malaysia, 43300 Serdang, Selangor, Malaysia

Full list of author information is available at the end of the article
}

which commonly associated with cognitive disability which can be revealed by magnetic resonance imaging (MRI) [6]. It was established that lesion load measurement on brain could predict neurological disorders occurred during several years. Currently, in new MS diagnosis criterion, the presence of a single cortical lesion on a cognitive syndrome indicates that the patient is suspected to MS [7]. It has been validated that routine MRI techniques is not enough to detect and quantify cortical lesions load on MS patients [8, 9]. As previous studies reported, FLAIR technique is poor to identify the exact boundary between the cortex and subcortical white matter. So, leading to misinterpretation of lesions in respect to the exact location of the lesion whether they are juxtacortical or mixed white/gray mater [10]. But a 
number of study have recently reported using of the double inversion recovery (DIR) technique provides higher quality images with adequate sensitivity in discriminating white matter from the gray matter lesions $[11,12]$. Clinically, EDSS can be handled to evaluate the degree of neurologic damages and the rate of disability in patients with MS which is the most widely used method of evaluation nowadays. Previous studies suggested that a major advantage of DIR is the positive association between clinical outcomes and cerebral cortex lesions [13, 14].

Employing DIR sequence in diagnosis of neurological disorders has been suggested in recent years [7]. The potential of the DIR sequence over the conventional 2D FLAIR and T2W imaging sequences in detection of small size lesion in MS patients with an improved image contrast and its specificity in lesion detection encouraged us to assess the lesion load on MS patient among Saudi Arabian population using FLAIR, DIR, and T2 sequences.

\section{The aim of our study}

The aim of our study was to evaluate the lesion load among MS patients using DIR, FLAIR, and T2WI sequences in different anatomical regions. In addition, we studied the role of DIR sequence and its clinical correlation with expanded disability scoring system (EDSS) to demonstrate the importance of DIR sequence as a practical test for clinical disability.

\section{Methods}

In our retrospective study, 97 MS patients including 34 male and 63 females average age 20 and 49 years (mean = 31.44 and $\mathrm{SD}=6.86$ ) were confirmed by neurologist accordance with 2017 McDonald criteria, randomly selected from 2017 to 2019 in the major hospital in Saudi Arabia. Result of frequency analysis according to their clinical symptoms showed that the majority of patients $(n=87)$ demonstrated relapsing remitting multiple sclerosis (RRMS), 5 cases presented primary progressive (PPMS) and 5 cases with clinically isolated syndrome (CIS).

This study was approved by the local ethics committee of the hospitals, and all the patients filled out a written consent before the MRI exam.

All images were acquired using 3T scanner (Siemens Skyra) at the radiology department of the two major hospitals selected for the study in Saudi Arabia with a standard 16 channel head coil for brain imaging.

The images from the DIR, FLAIR, and T2 TSE sequence were compared on axial plane with identical anatomic position. The parameters are summarized on Table 1. In DIR sequence, two inversion delay times (TI1 $=3000$ and TI2 $=425 \mathrm{~ms}$ ) are embedded in the system. TI1 and TI2 clarify the intervals between the two
Table $1 \mathrm{MRI}$ sequence parameters for multiple sclerosis

\begin{tabular}{llll}
\hline Parameter & DIR & FLAIR & T2W1 \\
\hline Repetition time (ms) & 7500 & 9000 & 4300 \\
Echo time (ms) & 318 & 84 & 83 \\
Inversion time (ms) & $3000 / 425$ & 2500 & \\
Slice thickness (mm) & 1.40 & 4 & 4 \\
Field of view (mm) & 260 & 240 & 240 \\
Matrix & 192 & 320 & 320 \\
Voxel size & $1.4^{*} 1.4^{*} 1.4$ & $0.8^{*} 0.8^{*} 4$ & $0.8^{*} 0.8^{*} 4$ \\
Number of signal averaging (NSA) & 1 & 1 & 2 \\
\hline
\end{tabular}

$180^{\circ}$ invertor pulses and the $90^{\circ}$ excitation pulse. The inversion times allows to demonstrate the cortex or the white matter depends on CSF and white matter to be suppressed or CSF and gray matter, so, depicts gray matter or white matter subsequently. The prolonged TI in DIR (TI1 $=3000$ ) allows better suppression of cerebrospinal fluid (CSF) compared with the TI $=2500$ in FLAIR.

\section{Image acquisition and MRI interpretation}

The images were interpreted by certified radiologist with neuroradiological experience, blinded to the clinical presentation and the results of the paraclinical tests.

There was some high signal intensity in a striped shape known as flow artifacts in extra-cortical regions rising from the major vessels or sinuses which considered artifacts while MS lesions were identified as hyper intense spots observed with a size of $\geq 2 \mathrm{~mm}$ in contrast to these spots. Detected lesions were divided respecting to their anatomical locations into five categories including infratentorial, juxtacortical, subcortical, periventricular, and cortical. The mean values of the contrast were calculated among lesions/normal appearing grey matter (NAGM), lesions/ normal appearing white matter (NAWM), lesions/cerebrospinal fluid (CSF), and NAWM/NAGM separately in three sequences in divided anatomical areas based on (SI1_SI2)/(SI1 + SI2). In this equivalent, SI1 indicates lesions signal intensity, SI2 representing the signal intensity of the NAWM, NAGM, or CSF. The signal intensity (SI) was specified by placing the region-of-interest (ROI) in a mean size of $3 \mathrm{~mm} \times 3$ $\mathrm{mm}$ on the lesions, the NAWM, and NAGM in all anatomical regions. All the patients were clinically evaluated with expanded disability status scale (EDSS) before the MRI exam commenced.

\section{Statistical analysis}

Statistical analysis was done using IBM SPSS statistics software version 24.0. The data analysis was expressed using descriptive statistic. To evaluate the research hypotheses, inferential statistic such as Pearson/Spearman correlation coefficient, one-way repeated measure 
ANOVA, and paired-sample $t$ tests were applied for difference of three methods if data were distributed normally. Non-parametric method for comparison such as Friedman and Wilcoxon-test and Spearman correlation to determine association between researches variables were applied on the data that did not meet with the assumption of normal distribution.

\section{Results}

Clinical symptoms among patients indicated that the most frequent symptom was numbness (46.4\%), visual disorders (40.2\%), and muscle weakness (40.2\%); the lowest frequency belonged to headache $(12.4 \%)$ and $28.9 \%$ with other symptoms such as sphincteric dysfunction. Patients with suboptimal image quality and other neurological disorders were excluded.

The total number of lesions were finally calculated and compared among three sequences. The average total number of lesions among patients in all region based on DIR was $M=37.67$, FLAIR was $M=29.57$, and for T2WI was $M=27.47$ (ranged 2 to 101). Results of Friedman's analysis of variance by ranks (Table 2 ) showed a significant difference among three sequences $\left(R^{2}=\right.$ 88.275, $p<0.01$ ).

The average number of lesions in infratentorial region based on DIR was $M=3.84$, for FLAIR $M=2.31$ and for T2WI was $M=2.87$. There was a significant difference among three sequences $\left(R^{2}=54.256, p<0.01\right)$ with significantly highest number of lesions belonged to DIR and also T2WI significantly higher than FLAIR imaging Fig. 1.

In juxtacortical region DIR showed a significant difference among three method $\left(R^{2}=44.319, p<0.01\right)$, and the average number of lesions was $M=5.75$, FLAIR was $M=4.43$ and for T2WI, $M=4.15$.

DIR sequence indicated the highest number of lesions $\left.R^{2}=44.319, p<0.01\right)$ with the mean number of lesion $M=15.07$ in subcortical and FLAIR $(M=12.79)$ was significantly higher than T2WI $(M=11.52)$ as well.

In the periventricular region, a significant difference was observed by DIR $\left(R^{2}=58.617, p<0.01\right)$ with $M=$ 10.66, FLAIR was $M=8.75$ and for T2WI was $M=$
8.32, and also, FLAIR was significantly better than T2WI Figs. 2 and 3. DIR exhibited significantly higher number of lesions in cortical region $\left(R^{2}=72.456, p<\right.$ $0.01)$ regarding to FLAIR which was significantly higher than T2WI Fig. 4. The mean number of lesions in cortical region based on DIR was $M=2.35$, for FLAIR was $M=1.29$ and for T2WI, $M=0.62$. The lesion load measurements shown on Table 2.

All patients went through neurological examination by a neurologist at the time of MRI to assess their functional capacities by means of EDSS according to the 2017 revised Mc Donald criteria.

The results of our study found a significant positive association between infratentorial lesions and higher EDSS score or clinical disability. Our results displayed the highest, significant and strong correlation between the number of detected lesions and EDSS by DIR sequence $(r=0.584, p<0.001)$ in infratentorial region. In connection with cortical lesions, our findings indicated a positive and significant results between the number of lesions and EDSS by DIR sequence $(r=0.273, p=0.007)$ in cortical region, while relationship was almost weak.

\section{Image contrast measurement}

The contrast measurements of DIR, FLAIR, and T2W TSE imaging are listed in Table 3.

The contrast ratio indicated that the highest contrast ratio in infratentorial belonged to DIR which was significantly higher than other sequences $\left(R^{2}=120.114, p<\right.$ 0.01 ) followed by T2WI which was significantly higher than FLAIR Fig. 1. In supratentorial including juxtacortical, subcortical and periventricular there were the highest contrast ratio difference among three sequences $\left(R^{2}\right.$ $=128.878,\left(R^{2}=152.271\right.$, and $R^{2}=167.558$ respectively with $p<0.001)$. The significantly highest contrast ratio in these three regions belonged to DIR and T2WI was significantly greater than FLAIR.

FLAIR showed significantly higher contrast ratio between CSF and lesion $\left(R^{2}=155.881, p<0.001\right)$ and DIR was significantly higher than T2WI.

A significant difference of contrast ratio between Lesion/NAGM were observed $\left(R^{2}=22.041, p<0.001\right)$ by

Table 2 Comparison of number of lesions based on regions among DIR, FLAIR and T2WI

\begin{tabular}{|c|c|c|c|c|c|c|c|c|c|c|}
\hline \multirow[t]{2}{*}{ Regions } & \multicolumn{2}{|l|}{ DIR } & \multicolumn{2}{|l|}{ FLAIR } & \multicolumn{2}{|l|}{$\mathrm{T} 2 \mathrm{WI}$} & \multirow{2}{*}{$\begin{array}{l}\text { Relative } \\
\text { gain } \\
\text { DIR/FLAIR }\end{array}$} & \multirow{2}{*}{$\begin{array}{l}\text { Relative } \\
\text { gain } \\
\text { DIR/T2WI }\end{array}$} & \multirow[t]{2}{*}{$R^{2}$} & \multirow{2}{*}{$\begin{array}{l}p \\
\text { value }\end{array}$} \\
\hline & $\begin{array}{l}\text { Mean } \\
\text { (median) }\end{array}$ & $\begin{array}{l}\text { SD } \\
\text { (IQR) }\end{array}$ & $\begin{array}{l}\text { Mean } \\
\text { (median) }\end{array}$ & $\begin{array}{l}\text { SD } \\
\text { (IQR) }\end{array}$ & $\begin{array}{l}\text { Mean } \\
\text { (median) }\end{array}$ & $\begin{array}{l}\text { SD } \\
\text { (IQR) }\end{array}$ & & & & \\
\hline Infratentorial & $3.84(2)^{a}$ & $4.05(5)$ & $2.31(2)^{c}$ & $2.78(3)$ & $2.87(2)^{b}$ & $3.32(3)$ & $66.23 \%$ & $33.80 \%$ & $54.265^{*}$ & $<0.001$ \\
\hline Juxtacortical & $5.75(4)^{\mathrm{a}}$ & $5(9)$ & $4.43(3)^{\mathrm{b}}$ & $3.91(5)$ & $4.15(3)^{b}$ & $3.95(7)$ & $29.80 \%$ & $38.55 \%$ & $44.319^{*}$ & $<0.001$ \\
\hline Subcortical & $15.07(15)^{\mathrm{a}}$ & $9.39(15)$ & $12.79(13)^{b}$ & $7.75(11)$ & $11.52(11)^{c}$ & $8.13(10)$ & $17.83 \%$ & $30.82 \%$ & $67.192^{*}$ & $<0.001$ \\
\hline Periventricular & $10.66(8)^{a}$ & $7.51(12)$ & $8.75(6)^{b}$ & $6.49(10)$ & $8.32(5)^{c}$ & $7.08(10)$ & $21.83 \%$ & $28.13 \%$ & $58.617^{*}$ & $<0.001$ \\
\hline Cortical & $2.35(2)^{a}$ & $2.72(4)$ & $1.29(1)^{b}$ & $2.3(2)$ & $0.62(0)^{c}$ & $1.39(1)$ & $82.17 \%$ & $279.03 \%$ & $72.456^{*}$ & $<0.001$ \\
\hline
\end{tabular}

*Significant at 0.05 level based on adjusted by the Bonferroni correction for multiple tests. Medians with different letter are significantly different 


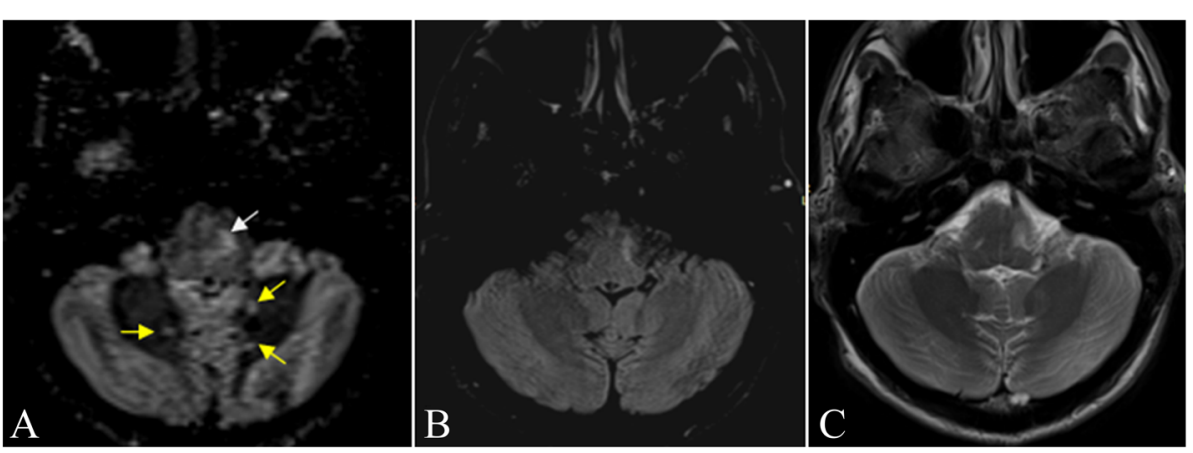

Fig. 1 Axial MRI of brain at the level of medulla oblongata. A hyper-intense lesion observed in the left aspect of medulla oblongata with better delineation on DIR (a) compared to FLAIR (b), and T2WI (c). Also, several small hyper-intense juxtacortical lesions (yellow arrows) are seen involving bilateral cerebellar white matter that are identified on DIR (a) and not seen on both FLAIR (b) and T2WI image (c)

DIR and T2WI with no statistical difference between them, but were significantly higher than FLAIR. The results indicated the highest contrast ratio between NAGM/NAWM $\left(R^{2}=156.545, p<0.001\right)$ belonged to DIR, also, T2WI was significantly greater than FLAIR.

\section{Discussion}

Multiple sclerosis is a disease generally has been considered as white matter disorder. Recently, the increasing number of gray matter involvement has been proved by pathophysiological reviews in MS patients [15, 16].

An obvious histopathological variation, particularly in patients with primary progressive or secondary progressive MS is cortical demyelination, so it is required to demonstrate cortical and subcortical lesions by different pulse sequences $[5,17]$.

Although conventional MRI sequences are not successful in cortical lesions demonstration, and despite the advances in new MR techniques such as magnetic resonance spectroscopy (MRS) and diffusion tensor imaging (DTI), still conventional MRI protocols playing an important role in early diagnosis of MS or CIS (suggestive of MS) [4-6].

Previous study stated MS plaques better displayed in axial plane in the area of subcortical and juxtacortical, while the sagittal plane is appropriate to demonstrate MS lesions in collo-septal and corpus callosum regions [13, 18]. T2-weighted TSE sequences are more sensitive to detect infratentorial lesions compared to FLAIR imaging sequences and FLAIR sequences have adverse results in the evaluation of the infratentorial parenchyma which it may be due to the differences in relaxation times compared to the supratentorial parenchyma [19-21]. Whereas, FLAIR has the highest sensitivity in the supratentorial area, near the CSF such as juxtacortical and the periventricular white matter. The signal from CSF will be suppressed in FLAIR and DIR pulse sequences while on the contrary, DIR also attenuates the signal from white matter (WM) and the sensitivity of DIR pulse sequence increases to detect brain lesions in WM and gray matter (GM) compared to sequences such as proton density (PD) or T2WI and T1weighted (T1W) MR sequences at 1.5 and $3.0 \mathrm{~T}[18,22]$.
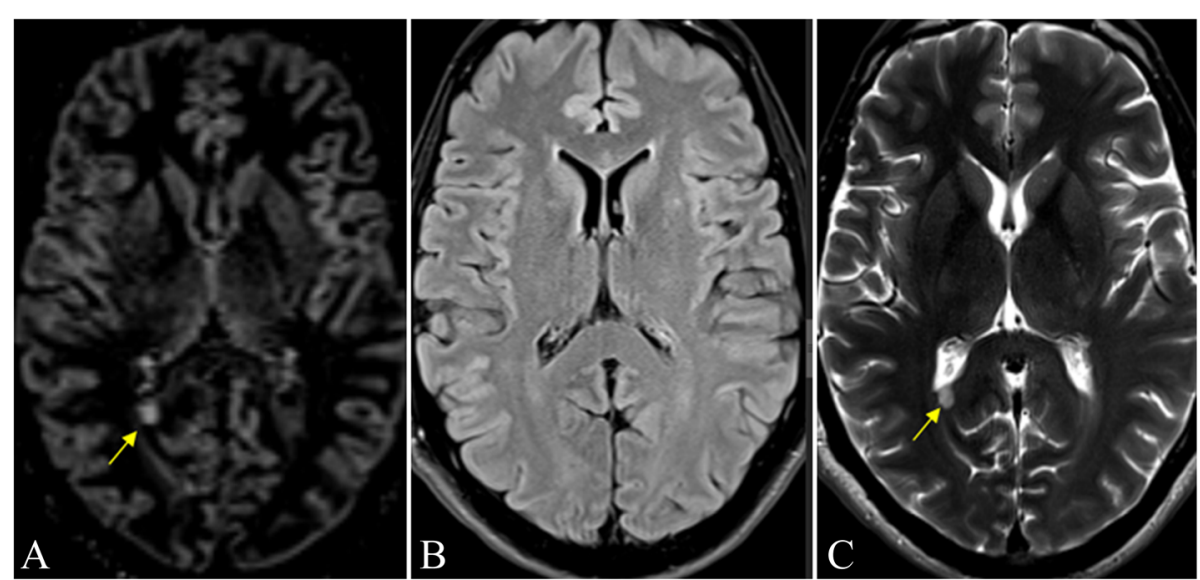

Fig. 2 Axial MRI of the brain. A lesion (arrow) with higher signal intensity and greater contrast and enables improved visualization of the lesion in $\operatorname{DIR}(\mathbf{a})$ and T2WI (c) and not seen in FLAIR (b) 

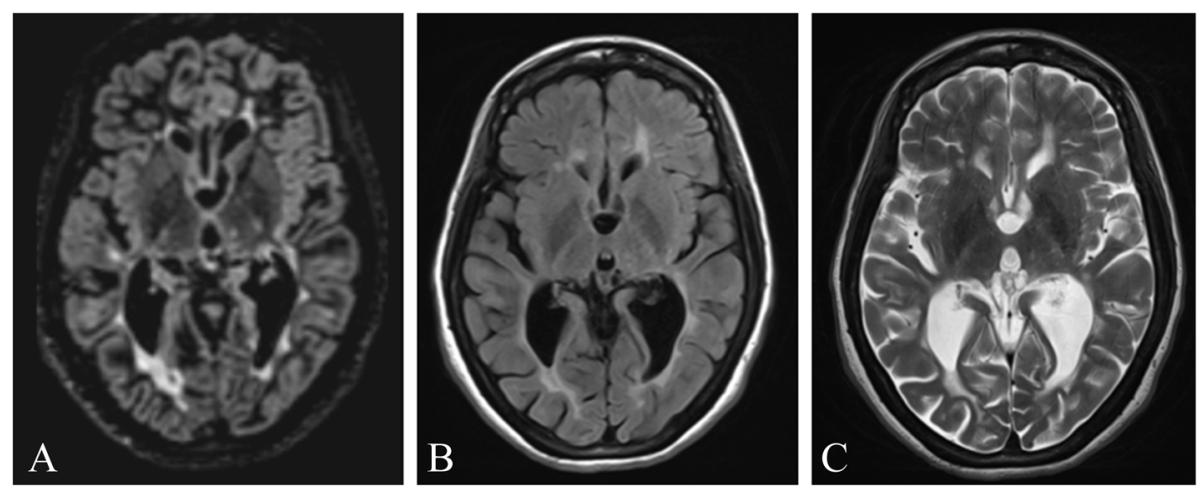

Fig. 3 Note the numerous confluent periventricular MS plaques in a RRMS patient with EDSS2 and numbness and dysfunction. DIR (a), FLAIR (b), and T2W_TSE (c). DIR image showing more counted lesions with sharply defined borders compared to FLAIR and T2W_TSE

DIR has potential advantages over conventional MRI protocols to demonstrate both infratentorial and supratentorial MS lesions and also provides improved appearance of early lesions [20, 23]. Various pulse sequences are used recently to enhance the sensitivity of MRI to identify more MS plaques. The difference T1 relaxation times between GM/CSF and GM/WM results in optimized contrast between gray and white matter and it demonstrates a great boundary of GM. DIR was developed by Redpath and Smith. It produces a dual contrast of FLAIR and short tau inversion recovery (STIR) [24, 25].

Two-dimensional (2D) and 3D FLAIR imaging have shown the increased displaying of cortical and subcortical lesions. FLAIR sequence is extremely sensitive to detect supratentorial MS lesions due to the CSF attenuation, especially for juxtacortical and periventricular white matter. But, unlike the DIR sequence, these sequences do not have this potential to determine the exact boundary between the cortex and subcortical WM clearly, cortical and juxtacortical lesions detection have improved beyond DIR sequence [24, 26]. Nowadays, numerous MR techniques such as MRS, DTI, and pseudocontinuous arterial spin labeling have been used to detect MS lesion and determine its relation to patient's disability [25, 27].

Recent studies have shown that the extent of gray matter damage is strongly correlated with the spread of the disease and the degree of physical disability and cognitive disorders $[28,29]$.

In a previous study, Chard [30] introduced the DIR sequence as a diagnostic criteria in MS patients, especially for the acute symptoms and gray matter injuries produced by new MS plaques in the cerebral cortex [30, 31]. Two different inversion pulses are set in DIR sequence, which remove the CSF and white matter as well, so it attains a greater delineation between gray and white matter $[18,31]$.

In our study, brain MRI was performed using conventional sequences as well as DIR, FLAIR, and T2W_TSE techniques. The number of MS plaques in each anatomical region and the total number of MS lesions were calculated separately in each sequence in all patients.

The result of our study was quite promising, DIR showed significantly higher total MS lesions load $(N=$ 3647) in all anatomical regions compared to FLAIR imaging $(N=2896)$ and T2W_TSE $(N=2697)$, our overall

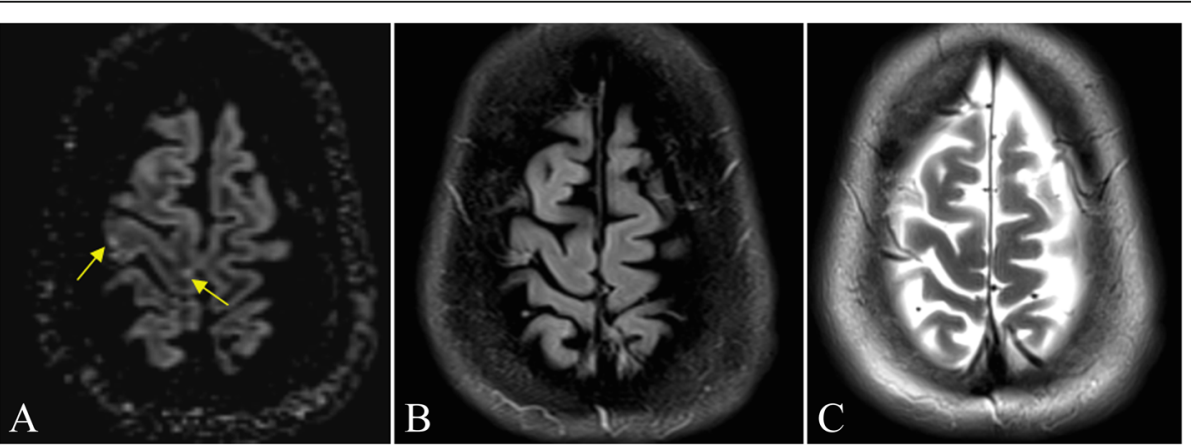

Fig. 4 Axial MRI of the brain at DIR (a), FLAIR (b), and T2WI (c) displaying two small hyper-intense cortical lesions on DIR sequence and not observed at corresponding FLAIR and T2W images 
Table 3 Comparison of Image Contrast based on regions among DIR, FLAIR, and T2WI

\begin{tabular}{|c|c|c|c|c|c|c|c|c|}
\hline \multirow[t]{2}{*}{ Regions } & \multicolumn{2}{|l|}{ DIR } & \multicolumn{2}{|l|}{ FLAIR } & \multicolumn{2}{|l|}{$\mathrm{T} 2 \mathrm{WI}$} & \multirow[t]{2}{*}{$R^{2}$} & \multirow{2}{*}{$\begin{array}{l}p \\
\text { value }\end{array}$} \\
\hline & Mean (median) & $\begin{array}{l}\text { SD } \\
\text { (IQR) }\end{array}$ & Mean (median) & $\begin{array}{l}\text { SD } \\
\text { (IQR) }\end{array}$ & Mean (median) & $\begin{array}{l}\text { SD } \\
\text { (IQR) }\end{array}$ & & \\
\hline Infratentorial & $0.58(0.56)^{a}$ & $0.16(0.24)$ & $0.16(0.17)^{c}$ & $0.16(0.09)$ & $0.2(0.26)^{\mathbf{b}}$ & $0.26(0.12)$ & $120.114^{*}$ & $<0.001$ \\
\hline Juxtacortical & $0.68(0.71)^{a}$ & $0.21(0.12)$ & $0.23(0.23)^{c}$ & $0.06(0.1)$ & $0.31(0.32)^{\mathbf{b}}$ & $0.12(0.16)$ & $128.878^{*}$ & $<0.001$ \\
\hline Subcortical & $0.68(0.69)^{a}$ & $0.14(0.15)$ & $0.28(0.26)^{c}$ & $0.12(0.11)$ & $0.38(0.37)^{\mathbf{b}}$ & $0.14(0.18)$ & $152.271^{*}$ & $<0.001$ \\
\hline Periventricular & $0.73(0.72)^{\mathbf{a}}$ & $0.08(0.1)$ & $0.3(0.31)^{c}$ & $0.15(0.11)$ & $0.42(0.41)^{\mathbf{b}}$ & $0.11(0.18)$ & $167.558^{*}$ & $<0.001$ \\
\hline CSF & $0.87(0.89)^{\mathbf{b}}$ & $0.07(0.1)$ & $0.92(0.92)^{a}$ & $0.04(0.07)$ & $-0.33(-0.36)^{c}$ & $0.13(0.18)$ & $155.881^{*}$ & $<0.001$ \\
\hline NAGM & $0.19(0.2)^{\mathbf{a}}$ & $0.13(0.18)$ & $0.12(0.13)^{\mathbf{b}}$ & $0.1(0.1)$ & $0.18(0.19) a$ & $0.16(0.24)$ & $22.041^{*}$ & $<0.001$ \\
\hline NAGM/NAWM & $0.58(0.59)^{a}$ & $0.13(0.16)$ & $0.15(0.14)^{c}$ & $0.11(0.10)$ & $0.24(0.23)^{\mathbf{b}}$ & $0.08(0.09)$ & $156.545^{*}$ & $<0.001$ \\
\hline
\end{tabular}

*Significant at 0.05 level median with different letter are significantly different

findings were in agreement with the findings reported in different studies of Abidi et al. [18], Umino et al. [17], Prosperini et al. [32], and Hickman et al. [33].

With respect to infratentorial region, DIR identified increased number of lesions in cerebellum and brain stem with better delineation, even compared with the T2W_TSE which still considered as a gold sequence at this region. These findings were also in agreement with the findings reported by Abidi et al. [18], Umino et al. [17], Prosperini et al. [32], and Hickman et al. [33].

A noticeably higher number of lesions seen in juxtacortical white matter, these findings were in agreement with those reported from Abidi et al. [18] and Hickman et al. [33] but, Umino et al. [17] reported a slightly lower number of detected lesions in this region, they noted slightly more lesions in the juxtacortical with FLAIR imaging. And also, Prosperini et al. [32] noted statistically significant increase in juxtacortical and mixed WM/GM lesions detection on DIR imaging compared to FLAIR and T2W_TSE.

Abidi et al. [18], Umino et al. [17], Prosperini et al. [32], and Hickman et al. [33], differed in their findings from our study as they reported a lower number of MS lesions in deep white matter with the DIR and slightly higher number of lesions at this region by FLAIR imaging sequence.

DIR revealed significantly higher mean number of lesions in the periventricular WM compared with both FLAIR and T2W_TSE, but there was no significant difference between the mean number of lesions between FLAIR and T2W_TSE at this region.

Abidi et al. [18], Hamed et al. [13], and Wattjes et al. [20] reported a slightly increasing number of lesions regarding to FLAIR but statistically difference for T2W imaging at periventricular region.

By using the DIR sequence, 64 cases of 97 cases in our study were found to have cortical lesions as well as white matter lesions, while FLAIR displayed the lesions in 48, and T2W indicated them in 26 cases in the cortex. By DIR imaging radiologist could differentiate the exact location of the lesion whether the lesion was pure cortical or mixed WM/GM or juxtacortical.

Our main interest at this study was to identify gray matter lesions in patients who are affected by MS disease and better detected by DIR sequence. MR imaging with 3D DIR enabled markedly increasing number of intracortical lesions $(N=231)$ compared to FLAIR $(N=$ $130)$ and T2WI $(N=72)$ Fig. 4 . Our results were consistent with the findings of the study done by Abidi et al. [18], Prosperini et al. [32], and Hickman et al. [33].

In connection with the number of lesions and EDSS score in different anatomic regions, our study with increased number of lesions in infratentorial and higher EDSS score is worth discussing.

It is important to diagnose infratentorial lesions because these findings indicate long-term disability in patients with MS which are along with early detection of the disease [23]. Performing DIR sequence is a beneficial method which may affect the diagnosis and deciding the treatment at the onset of the disease or especially in patients with CIS. The number of lesions in infratentorial area is an important factor to predict the disease with long-term disability throughout the patient's life. So, appropriate treatment decisions increases by higher diagnostic accuracy of these lesions and it has a significant effect to decrease the long-term disability in patients with MS [20, 27].

The specific structure of the infratentorial region regarding to high density of neural fibers in this region $[32,33]$ can explain importance of our results and the correlation between clinical outcomes and MRI findings in the infratentorial region more strongly than other areas of the brain.

Two employed inversion pulses improve the detection of cortical lesions on DIR sequence by nulling the signal from CSF and white matter as well. Subsequently, this double suppression design produces a lower SNR image. The lower SNR as well as the greater tendency to pulsation artifacts than FLAIR and T2_TSE should be considered. Also, previous studies pointed out non-uniform 
signal of the magnetic field in the cortex of the limbic region, as well as the cortex of the central salcus. They indicated lower SNR and higher scanning time prevents the high spatial resolution requiring to display the cortex with a thickness of only 2 to $4 \mathrm{~mm}$ [23]. However, the CSF nulling was appropriate in our images obtained by DIR sequence even in the worst conditions.

The hyper intense artifacts seen in DIR are ribbon like and characterized by their bilateral prevalence and symmetrical shape, which are characterized by deformations in successive segments and distinguished in successive slices. The observation of their variable appearance in several consecutive slices and other MRI sequences including T1-weight or FLAIR can help to distinguish them as lesions with irregulated shape or as artifacts caused by rounded cerebral cortical vessels. Artifacts usually occur in posterior fossa, choroid plexus, periventricular WM, periaqueductal, and brainstem tissue appear to be as a result of trans ependymal CSF effusion and pulsation, cerebral sinuses or larger vessels. So, the greater the extent of the artifacts at the cortico-sulcal interface, the greater likelihood of counting misinterpreted cortical lesions. Therefore, a significant reduction of such artifacts will increase the quality of the diagnosis $[18,34,35]$.

In our study, an acquainted radiologist with these artifacts paid careful attention to exclude artifacts seen in the areas such as the anterior temporal lobes, occipital lobes, insula. and medial frontal lobes. It helped to eliminate cortical vessels not be counted falsely as lesions. There were very few cerebrospinal fluid and vessels artifact by using 3D DIR sequence and higher magnetic field strength comparing with the 2D FLAIR. So, despite the higher acquisition time, we were encouraged to do 3D DIR sequence because of the advantage of the 3D sequence in the lower artifact display.

The various benefits of 3D scans over their identical 2D sequences have encouraged us to do 3D DIR sequence. Having isotropic voxels with equal length in all three directions, are the most important advantages of images obtained from 3D scans and there is no need to scan complementary planes such as separate sagittal and axial. Since there are no gaps between the slices, so 3D scans can easily keep the image quality in term of contrast-to-noise ratio (CNR) providing greater spatial resolution. Three-dimensional MRI sequences, due to the use of smaller slice thicknesses, detect smaller size MS plaques especially cortical lesions more precisely and less flow artifacts compared to two-dimensional sequences [34, 36].

There are no artifacts of blood flow and CSF in 3-D sequences because of non-selective inversion pulses. Three-dimensional MRI is crucial for the diagnosis of cerebral atrophy and evaluation of the cortical lesions load that cause more cognitive impairment and physical disability than white matter $[34,35]$.

\section{Conclusion}

The 3D DIR sequence at 3T provide faster acquisition time and higher sensitivity in detection of pure intracortical lesions compared with conventional brain MRI sequences. Since cortical lesions produces cognitive disorders and physical dysfunction thus, a significant attention should be paid in detection of even a single new lesion in gray matter. Therefore, we strongly recommend the addition of DIR imaging technique as a routine brain MRI sequence.

\section{Abbreviations}

DIR: Double inversion recovery; FLAIR: Fluid attenuated inversion recovery; MS: Multiple sclerosis; WM: White matter; GM: Gray matter; NAGM: Normal gray matter; NAWM: Normal white matter; EDSS: Expanded Disability Status Scale; T: Tesala; T1: T1-weighted imaging; T2W1: T2-weighted imaging; MRI: Magnetic resonance imaging; 3D: Three dimension; CSF: Cerebrospinal fluid; RRMS: Demonstrated relapsing remitting multiple sclerosis;

PPMS: Presented primary progressive; CIS: Clinically isolated syndrome; ROI: Region-of-interest; PD: Proton density

\section{Acknowledgements}

The authors wish to acknowledge the support of the Radiology Unit, Department of Radiology, Prince Mohammed Hospital, Saudi Arabia, and Universiti Putra Malaysia for their assistance during the analysis.

\section{Declaration}

The research meets all applicable standards with regard to the ethics of experimentation and research integrity, and the following is being certified/ declared true. As an expert scientist and along with co-authors of concerned field, the paper has been submitted with full responsibility, following due ethical procedure, and there is no duplicate publication, fraud, plagiarism, or concerns about animal or human experimentation, with full consent for publication.

\section{Authors' contributions}

ADA, HAH, SS, and RM made substantial contributions to the conception. ADA and OIA helped in the acquisition and analysis. ADA and RM carried out the neurological assessment of the cases. ADA, AA, HA, and RM helped in the interpretation of data; ADA drafted the work and $\mathrm{HAH}, \mathrm{SS}$, and RM substantively revised the manuscripts and approved the submitted version and also agreed both to be personally accountable for the author's own contributions and to ensure that questions related to the accuracy or integrity of any part of the work, even ones in which the author was not personally involved, are appropriately investigated, resolved, and the resolution documented in the literature. All author(s) read and approved the final manuscript.

\section{Funding}

No funding was obtained for this study.

\section{Availability of data and materials}

The datasets used and/or analyzed during the current study are available with the corresponding author (Prof. Rozi Mahmud) on reasonable request.

\section{Ethics approval and consent to participate}

Written informed consent was obtained from the study participants or their representative prior to enrolment. The study was approved by the research ethics committee of the Faculty of Medicine and Health Sciences, Universiti Putra Malaysia, on 30th January, 2018, with a reference approval number: UPM/TNCPI/RMC/KEUPM/1.4.18.2 (JKEUPM) and from the Ministry of Health, Saudi Arabia with an approval number of IRB-148 on 1/8/1439AH. 


\section{Consent for publication}

All patients included in this research gave a written informed consent to publish the data contained within this study. However, where the patient is less than 16 years old or unconscious when consent for publication is requested, written informed consent for the publication of the data was given by their parent or legal guidance

\section{Competing interests}

The authors declare that they have no competing interests. However, none of the authors of this paper has a financial or personal relationship with other people or organizations that could inappropriately influence or bias the content of the paper.

\section{Author details}

${ }^{1}$ Department of Imaging, Faculty of Medicine and Health Sciences, Universiti Putra Malaysia, 43300 Serdang, Selangor, Malaysia. ${ }^{2}$ College of Applied Medical Sciences, King Saudi University, Riyadh, Saudi Arabia. ${ }^{3}$ Department of Radiology, Prince Mohammed Hospital, Riyadh, Saudi Arabia. ${ }^{4}$ Saudi Arabia Ministry of Health, Riyadh, Saudi Arabia.

\section{Received: 25 May 2020 Accepted: 8 September 2020}

Published online: 13 October 2020

\section{References}

1. Huang WJ, Chen WW, Zhang X (2017) Multiple sclerosis: pathology, diagnosis and treatments. Experimental and Therapeutic Medicine 13(6): 3163-3166

2. Kargarfard M, Shariat A, Ingle L, Cleland JA, Kargarfard M (2018) Randomized controlled trial to examine the impact of aquatic exercise training on functional capacity, balance, and perceptions of fatigue in female patients with multiple sclerosis. Arch Phys Med Rehabil 99(2):234-241

3. Sarbu N, Shih RY, Jones RV, Horkayne-Szakaly I, Oleaga L, Smirniotopoulos JG (2016) White matter diseases with radiologic-pathologic correlation. Radiographics. 36(5):1426-1447

4. Zahiri N, Abollahi I, Nabavi SM, Ehsani F, Arab AM, Shaw I et al (2017) Interference effect of prior explicit information on motor sequence learning in relapsing-remitting multiple sclerosis patients. The Malaysian journal of medical sciences: MJMS 24(1):69

5. Haider L, Simeonidou C, Steinberger G, Hametner S, Grigoriadis N, Deretzi G et al (2014) Multiple sclerosis deep grey matter: the relation between demyelination, neurodegeneration, inflammation and iron. J Neurol Neurochir Psychiatr 85(12):1386-1395

6. Narayanan L, Murray AD (2016) What can imaging tell us about cognitive impairment and dementia? World journal of radiology 8(3):240

7. Klawiter EC. Current and new directions in MRI in multiple sclerosis. Continuum: Lifelong Learning in Neurology. 2013;19(4 Multiple Sclerosis):1058.

8. Miller DH. Magnetic resonance imaging in multiple sclerosis: an overview. Multiple Sclerosis Therapeutics: CRC Press; 2019. p. 114-30.

9. Filippi M, Preziosa P, Banwell BL, Barkhof F, Ciccarelli O, De Stefano $\mathrm{N}$ et al (2019) Assessment of lesions on magnetic resonance imaging in multiple sclerosis: practical guidelines. Brain J Neurol 142(7):1858-1875

10. Futatsuya K, Kakeda S, Yoneda T, Ueda I, Watanabe K, Moriya J, et al. Juxtacortical lesions in multiple sclerosis: assessment of gray matter involvement using phase difference-enhanced imaging (PADRE). Magnetic Resonance in Medical Sciences. 2016:mp. 2015-0099.

11. Arevalo O, Riascos R, Rabiei P, Kamali A, Nelson F (2019) Standardizing magnetic resonance imaging protocols, requisitions, and reports in multiple sclerosis: an update for radiologist based on 2017 Magnetic Resonance Imaging in Multiple Sclerosis and 2018 Consortium of Multiple Sclerosis Centers Consensus Guidelines. J Comput Assist Tomogr 43(1):1-12

12. Tommasin S, Giannì C, De Giglio L, Pantano P (2019) Neuroimaging techniques to assess inflammation in multiple sclerosis. Neuroscience. 403:4-16

13. Hamed W, Fathi W, Mahmoud W, Elhawary G (2019) Diagnostic accuracy of double inversion recovery in delineation of multiple sclerosis lesions and its clinical correlation with expanded disability scoring system. Egypt J Radiol Nucl Med 50(1):1-8

14. Sahraian MA, Eshaghi A (2010) Role of MRI in diagnosis and treatment of multiple sclerosis. Clin Neurol Neurosurg 112(7):609-615

15. Bø L, Vedeler CA, Nyland HI, Trapp BD, Mørk SJ (2003) Subpial demyelination in the cerebral cortex of multiple sclerosis patients. J Neuropathol Exp Neurol 62(7):723-732
16. Geurts JJ, Bö L, Pouwels PJ, Castelijns JA, Polman CH, Barkhof F (2005) Cortical lesions in multiple sclerosis: combined postmortem MR imaging and histopathology. Am J Neuroradiol 26(3):572-577

17. Umino M, Maeda M, li Y, Tomimoto H, Sakuma H (2019) 3D double inversion recovery MR imaging: Clinical applications and usefulness in a wide spectrum of central nervous system diseases. J Neuroradiol 46(2):107-116

18. Abidi Z, Faeghi F, Mardanshahi Z, Mortazavi H (2017) Assessment of the diagnostic accuracy of double inversion recovery sequence compared with FLAIR and T2W_TSE in detection of cerebral multiple sclerosis lesions. Electron Physician 9(4):4162

19. Simon J, Li D, Traboulsee A, Coyle P, Arnold D, Barkhof F et al (2006) Standardized MR imaging protocol for multiple sclerosis: Consortium of MS Centers consensus guidelines. Am J Neuroradiol 27(2):455-461

20. Wattjes M, Lutterbey G, Gieseke J, Träber F, Klotz L, Schmidt S et al (2007) Double inversion recovery brain imaging at 3T: diagnostic value in the detection of multiple sclerosis lesions. Am J Neuroradiol 28(1):54-59

21. Polman $\mathrm{CH}$, Reingold SC, Banwell B, Clanet M, Cohen JA, Filippi M et al (2011) Diagnostic criteria for multiple sclerosis: 2010 revisions to the McDonald criteria. Ann Neurol 69(2):292-302

22. Klaver R, De Vries HE, Schenk GJ, Geurts JJ (2013) Grey matter damage in multiple sclerosis: a pathology perspective. Prion. 7(1):66-75

23. Wang KY, Uribe TA, Lincoln CM (2018) Comparing lesion detection of infratentorial multiple sclerosis lesions between T2-weighted spin-echo, 2DFLAIR, and 3D-FLAIR sequences. Clin Imaging 51:229-234

24. Elnekeidy AM, Kamal MA, Elfatatry AM, Elskeikh ML (2014) Added value of double inversion recovery magnetic resonance sequence in detection of cortical and white matter brain lesions in multiple sclerosis. The Egyptian Journal of Radiology and Nuclear Medicine 45(4):1193-1199

25. Vural G, Keklikoğlu H, Temel \$̧, Deniz O, Ercan K (2013) Comparison of double inversion recovery and conventional magnetic resonance brain imaging in patients with multiple sclerosis and relations with disease disability. Neuroradiol J 26(2):133-142

26. Geurts JJ, Pouwels PJ, Uitdehaag BM, Polman CH, Barkhof F, Castelijns JA (2005) Intracortical lesions in multiple sclerosis: improved detection with 3D double inversion-recovery MR imaging. Radiology. 236(1):254-260

27. Ertan G, Düz Arıcı Ö, Ulus S, Metin B (2018) Efficiency of double ınversion recovery (dır) sequence in the evaluation of supratentorial cortical lesions in multiple sclerosis

28. Sanfilipo MP, Benedict RH, Weinstock-Guttman B, Bakshi R (2006) Gray and white matter brain atrophy and neuropsychological impairment in multiple sclerosis. Neurology. 66(5):685-692

29. De Stefano N, Matthews P, Filippi M, Agosta F, De Luca M, Bartolozzi M et al (2003) Evidence of early cortical atrophy in MS: relevance to white matter changes and disability. Neurology. 60(7):1157-1162

30. Chard D (2014) Cortical lesion counts by double inversion recovery should be part of the MRI monitoring process for all MS patients: no. Mult Scler J 20(5):539

31. Turetschek $K$, Wunderbaldinger $P$, Bankier AA, Zontsich T, Graf O, Mallek R et al (1998) Double inversion recovery imaging of the brain: initial experience and comparison with fluid attenuated inversion recovery imaging. Magn Reson Imaging 16(2):127-135

32. Prosperini $L$, Kouleridou A, Petsas N, Leonardi L, Tona F, Pantano P et al (2011) The relationship between infratentorial lesions, balance deficit and accidental falls in multiple sclerosis. J Neurol Sci 304(1-2):55-60

33. Hickman S, Brierley C, Silver N, Moseley I, Scolding N, Compston D et al (2001) Infratentorial hypointense lesion volume on T1-weighted magnetic resonance imaging correlates with disability in patients with chronic cerebellar ataxia due to multiple sclerosis. J Neurol Sci 187(1-2):35-39

34. Eichinger P, Hock A, Schön S, Preibisch C, Kirschke JS, Mühlau M et al (2019) Acceleration of double inversion recovery sequences in multiple sclerosis with compressed sensing. Investig Radiol 54(6):319-324

35. Calabrese M, De Stefano N, Atzori M, Bernardi V, Mattisi I, Barachino L et al (2007) Detection of cortical inflammatory lesions by double inversion recovery magnetic resonance imaging in patients with multiple sclerosis. Arch Neurol 64(10):1416-1422

36. Khangure $S$, Khangure M (2011) MR imaging in multiple sclerosis: the accuracy of 3D double inversion recovery at 3 Tesla and the potential for single sequence imaging. Neuroradiol J 24(1):92-99

\section{Publisher's Note}

Springer Nature remains neutral with regard to jurisdictional claims in published maps and institutional affiliations. 